Case

\title{
Cardiac Tamponade caused by Broken Sternal Wire after Pectus Excavatum Repair: A Case Report
}

\author{
Soo Hyun Lee, MD, Bum Sang Cho, MD, Sung Jin Kim, PhD, Seung Young Lee, MD,
} Min Ho Kang, MD, Gi Seok Han, MD, and Sang-Hoon Cha, PhD

\begin{abstract}
Complications of pectus excavatum surgery include pneumothorax, pleuritis, hemothorax, pericardial effusion, displacement of bar, pericarditis and cardiac injury, etc. This is the case of a 15-year-old boy with cardiac tamponade caused by pericarditis who had taken the operation for a pectus excavatum repair one year previously. The cause was a sternal wire which was used for attachment of the bar to sternum that had fractured and migrated through the pericardium causing a pericardial injury and a pericarditis.
\end{abstract}

Keywords: cardiac tamponade, pectus excavatum, substernal Nuss bar, Ravitch repair, sternal wire

\section{Introduction}

Pectus excavatum is a congenital deformity caused by a depressed sternum and lower costal cartilage. ${ }^{1)}$ Various surgical methods using a metal bar have been reported to correct the deformity. However, there can be the adverse effect that the metal bar moves, and the bar is sometimes fixed with a string or a wire so that it cannot move. ${ }^{1-5)}$

It is quite rare that after pectus excavatum repair the fixing wire is broken, moves, and damages organs inside the pericardium or pericardium, and only 3 cases have been reported throughout the world. ${ }^{2-4)}$ As the authors experienced a case of pericarditis and pericardial tamponade caused by broken sterna wire after pectus excavatum repair, so we report the case along with literature review.

Department of Radiology, Chungbuk National University Hospital, Cheongju, Korea

Received: December 15, 2011; Accepted: January 17, 2012

Corresponding author: Bum Sang Cho, MD. Department of Radiology, Chungbuk National University Hospital, 410 Seongbong-ro, Heungduk-gu, Cheongju-si, Chungbuk 361-711, Korea

Email: sircircle@hanmail.net

(C)2012 The Editorial Committee of Annals of Thoracic and Cardiovascular Surgery. All rights reserved.

\section{Case Report}

A 15-year-old male patient visited the hospital for anterior chest pain aggravated suddenly on the day of the visit. The patient had surgery combing Ravitch repair and substernal Nuss bar fixing 14 months ago, and Nuss bar removal 3 months before the visit. As the skin incision was not conducted in the bar removal, the metal fixing wire in the sternum was not removed. Since then, there had been chest pain persistently. In physical examination on the visit, cyanosis, reduced heart sound, and increased jugular venous pressure were observed. Initial blood pressure was $100 / 60 \mathrm{mmHg}$, but it went down to $80 / 50$ $\mathrm{mmHg}$ in 10 minutes.

In the AP chest radiograph during the visit, cardiac silhouette was larger than before, and the shape of the fixing wire in the sternum was changed (Figs. 1 and 2). As pericardial effusion was suspected, we performed echocardiogram and confirmed the presence of pericardial effusion. Then, pericardial paracentesis was performed as an emergent measure, and $100 \mathrm{ml}$ of serous pericardial effusion was drained through a drainage tube. After hemodynamic stability had been restored, chest CT was performed in order to find the cause of pericardial effusion.

In chest $\mathrm{CT}$, a small amount of pericardial effusion remained and the wire connected to the sternum advanced inside the pericardium. In addition, pleural effusion was 


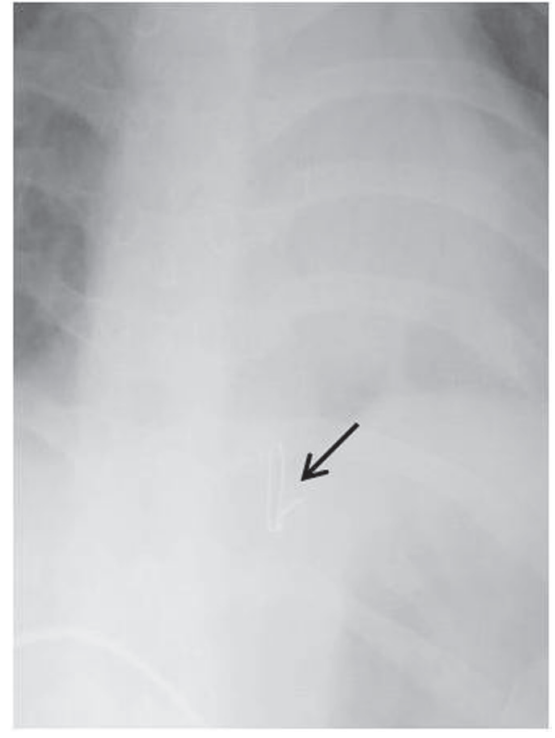

Fig. 1 Right after removal of Nuss bar, AP chest radiograph shows proper feature of sternal wire (arrow).

observed in both sides (Fig. 3).

The wire was removed through surgery. Then, the patient was admitted to the intensive care unit, and as the pain was eased and pericardial effusion drainage decreased he was discharged in 9 days. After 8 days, the patient made an outpatient visit for follow-up and did not show any abnormality in chest radiograph.

\section{Discussion}

Pectus excavatum means the backward recess of the sternum and costal cartilage adjacent to the sternum and occupies over $90 \%$ of congenital chest wall deformities. The cause has not been clarified, but is believed to happen as the growth of the sternum and the costal arch was impeded. ${ }^{1,2)}$ The surgical repair of pectus excavatum has been developed significantly during the last 50 years. Various techniques have been used successfully, and Ravitch procedure and Nuss procedure are known as the most representative surgical repair methods. ${ }^{1,2)}$ However, various methods can be used by the surgeon's subjective opinion, and in our case Ravitch procedure was used basically and, additionally, substernal fixing was performed after the insertion of a Nuss bar instead of a metal bar.

Known complications resulting from the surgery of pectus excavatum including the recurrence of pectus excavatum, pneumothorax, pleural effusion, pain, wound infection, displacement of the fixing bar, pericarditis,

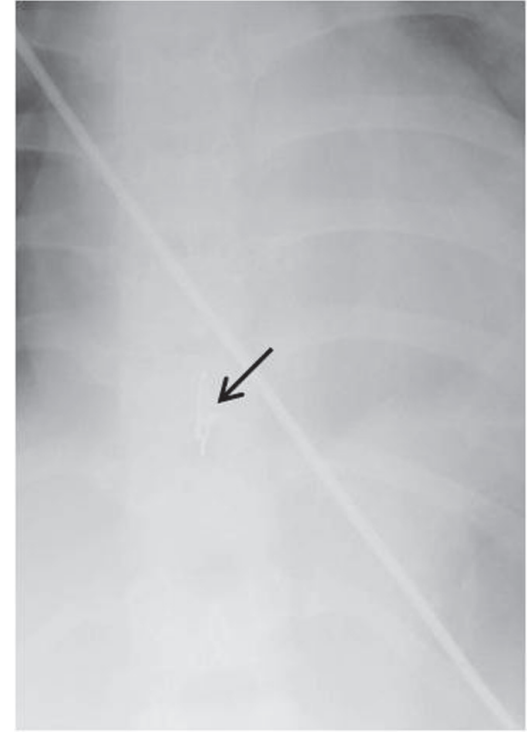

Fig. 23 months after removal of Nuss bar, AP chest radiograph shows fractured sternal wire (arrow).

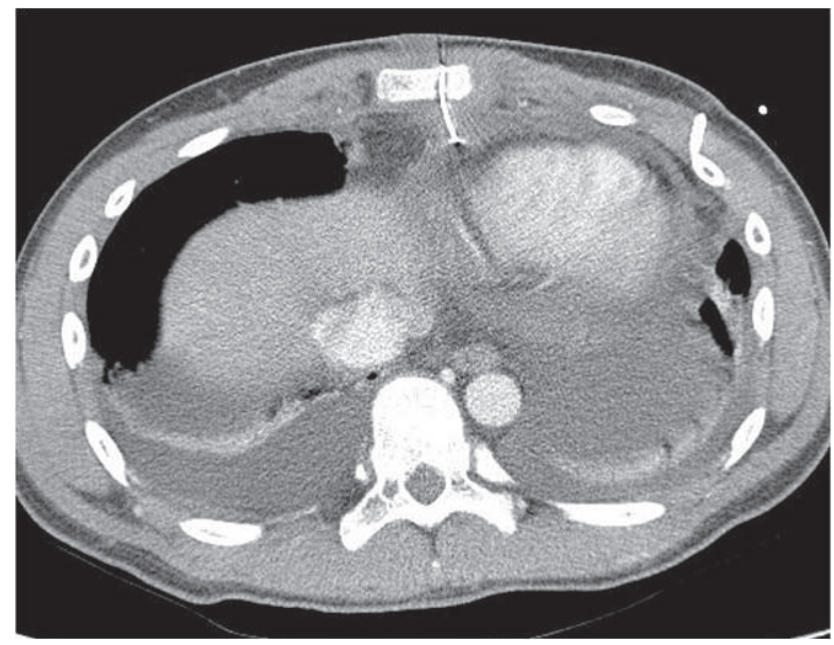

Fig. 3 Axial image of contrast enhanced chest CT shows fractured sternal wire penetrating the pericardium, small amount of pericardial effusion and both pleural effusion.

pericardial effusion, and cardiac injury. ${ }^{1-5)}$ Among them, pericarditis and pericardial effusion are rare complications and, in particular, such a case as this caused by the disconnection of fixing wire used in the surgery is extremely rare and only 2 cases have so far been reported throughout the world. ${ }^{3,4)}$ In our case, cardiac tamponade with pericarditis had developed by irritation of pericardium due to a broken sternal wire. We surmised that the accompanied bilateral pleural effusion was caused by pneumonia because the patient had symptoms such as 
cough and fever. The bilateral pleural effusion disappeared after 2 weeks of antibiotic therapy.

It is generally agreed that radiographic examination of the chest is extremely important, and its value is equally great in the diagnosis of disease of lung, mediastinum and bony thorax. The chest radiograph serves as a record of the presence or absence of disease on the date it was taken, and follow-up examinations can determine progress or development of disease. ${ }^{6}$ In our case, echocardiogram and CT were used for definite diagnosis, but putative diagnosis was made by chest radiograph that was performed for postoperative follow-up and confirmed the change of wire shape and the enlargement of the cardiac silhouette. Barakat et al. ${ }^{3)}$ and Cope et al. ${ }^{4)}$ reported cases of pericarditis and pericardial effusion, and pericardial tamponade caused by broken wire as in our case. However, the two cases were easier to diagnose than our case because chest radiograph showed changes not only in wire shape but also in its position. ${ }^{3,4)}$

In conclusion, when a patient who had pectus excavatum repair complains of chest pain, it should be considered that the wire used for fixing the metal bar may have broken and cause pain through irritating surrounding organs, and chest radiograph should be used to check the displacement or disconnection of the wire and cardiac silhouette. For patients with fixing wire in the sternum, moreover, careful attention should be paid in reading chest radiograph.

\section{Disclosure Statement}

We have no financial or other interest in the manufacture or distribution of the device.

\section{References}

1) Shamberger RC. Chest wall deformities. In: Shields TW, LoCicero J 3rd., Reed CE, eds. General thoracic surgery. Philadelphia: Lippincott Wiliams \& Wilkins, a Wolters Kluwer business, 2009; pp 599-613.

2) Jaroszewski D, Notrica D, McMahon L, et al. Current management of pectus excavatum: a review and update of therapy and treatment recommendations. J Am Board Fam Med 2010; 23: 230-9.

3) Barakat MJ, Morgan JA. Haemopericardium causing cardiac tamponade: a late complication of pectus excavatum repair. Heart 2004; 90: e22.

4) Cope SA, Rodda J. Cardiac tamponade presenting to the emergency department after sternal wire disruption. Emerg Med J 2004; 21: 389-90.

5) Mieno S, Ozawa H, Katsumata T. Ascending aortic injury caused by a fractured sternal wire 28 years after surgical intervention of pectus excavatum. J Thorac Cardiovasc Surg 2010; 140: e18-20.

6) John H. Juhl, Janet E. Methods of examination, technique, and anatomy of the chest. In: John H. Juhl, Andrew B. Crummy, Janet E, eds. Essentials of Radiologic Imaging. Philadelphia: Lippincott-Raven Publishers, 1998; pp 777-8. 